\title{
Novel A20-gene-eluting stent inhibits carotid artery restenosis in a porcine model
}

This article was published in the following Dove Press journal:

Drug Design, Development and Therapy

8 August 2016

Number of times this article has been viewed

\author{
Zhen-hua Zhou' \\ Jing Peng' \\ Zhao-you Meng' \\ Lin Chen' \\ Jia-Lu Huang' \\ He-qing Huang' \\ $\mathrm{Li} \mathrm{Li}^{2}$ \\ Wen Zeng ${ }^{2}$ \\ Yong $\mathrm{Wei}^{2}$ \\ Chu-Hong Zhu ${ }^{2}$ \\ Kang-Ning Chen' \\ 'Department of Neurology, \\ Cerebrovascular Disease Research \\ Institute, Southwest Hospital, \\ ${ }^{2}$ Department of Anatomy, Key \\ Laboratory for Biomechanics of \\ Chongqing, Third Military Medical \\ University, Chongqing, People's \\ Republic of China
}

Correspondence: Kang-Ning Chen Department of Neurology, Southwest Hospital, Third Military Medical

University, 30 Gao Tan Yan Road,

Chongqing 400038, People's Republic of China

Tel +86236875 4I54

Fax +862365460665

Email ckn640827@I26.com

Chu-Hong Zhu

Department of Anatomy, National \&

Regional Engineering Laboratory of

Tissue Engineering, Key Laboratory for

Biomechanics and Tissue Engineering

of Chongqing, Third Military Medical

University, 30 Gao Tan Yan Road,

Chongqing 400038, People's Republic

of China

Tel/fax +862368752225

Email zhuch99@yahoo.com
Background: Carotid artery stenosis is a major risk factor for ischemic stroke. Although carotid angioplasty and stenting using an embolic protection device has been introduced as a less invasive carotid revascularization approach, in-stent restenosis limits its long-term efficacy and safety. The objective of this study was to test the anti-restenosis effects of local stent-mediated delivery of the $A 20$ gene in a porcine carotid artery model.

Materials and methods: The pCDNA3.1EHA20 was firmly attached onto stents that had been collagen coated and treated with $N$-succinimidyl-3-(2-pyridyldithiol)propionate solution and anti-DNA immunoglobulin fixation. Anti-restenosis effects of modified vs control (the bare-metal stent and pCDNA3.1 void vector) stents were assessed by Western blot and scanning electron microscopy, as well as by morphological and inflammatory reaction analyses.

Results: Stent-delivered $A 20$ gene was locally expressed in porcine carotids in association with significantly greater extent of re-endothelialization at day 14 and of neointimal hyperplasia inhibition at 3 months than stenting without $A 20$ gene expression.

Conclusion: The A20-gene-eluting stent inhibits neointimal hyperplasia while promoting re-endothelialization and therefore constitutes a novel potential alternative to prevent restenosis while minimizing complications.

Keywords: restenosis, $A 20$, gene therapy, stent, endothelialization

\section{Introduction}

Ischemic stroke is a major cause of death and disability worldwide. ${ }^{1,2}$ Atherosclerotic stenosis of the carotid artery is a known major risk factor for ischemic stroke, and carotid angioplasty and stenting (CAS) has emerged as a potentially less invasive treatment alternative. Studies have suggested that CAS and carotid endarterectomy are associated with similar short- and long-term outcomes, ${ }^{3,4}$ and there is a growing evidence that the subgroup of patients aged $<70$ years may particularly benefit from CAS intervention. ${ }^{5,6}$

However, the occurrence of in-stent restenosis appears to limit the long-term efficacy and safety of CAS, and its occurrence is higher in intracranial atherosclerotic stenosis stenting. ${ }^{7,8}$

Unfortunately, fundamental research in the area of cerebrovascular ISR is very scarce presently.

Drug-eluting stents (DESs) have reduced the incidence of restenosis of the coronary artery $; 9,10$ however, meta-analyses of long-term outcomes have raised questions about their safety, ${ }^{11,12}$ including late-stage poor stent adherence to the vascular wall, longterm damage to endothelial cells (ECs), and late stent thrombosis. All these untoward effects are believed to result from DES-mediated nonselective dual inhibition of smooth muscle cells (SMCs) and ECs, leading to incomplete endothelialization. 
Compared with DES, gene-eluting stents can locally release biologically active agents to extend the agents' halflife at vascular lesions. ${ }^{13-15}$ Therefore, it is an intriguing strategy to use a stent as a platform for localized and prolonged gene therapy on vascular wall lesions. This strategy is designed to treat restenosis by targeting the molecular mechanisms underlying the pathology.

If rapid endothelialization could be achieved after interventional surgery for stent placement, it could significantly reduce the risk of late-stent thrombosis. In addition, endothelial regeneration can decrease intimal hyperplasia by inhibiting the proliferation and migration of SMCs. Thus, protecting the function of ECs plays an important role in preventing restenosis.

Recent studies have shown that the inflammatory response plays an important role in the genesis and development of restenosis. ${ }^{16,17}$ Histological and biochemical research has shown that platelets and white blood cells are activated following stent surgery, leading to a series of inflammatory responses. Therefore, the inflammatory response is also an important target for the prevention and treatment of restenosis.

The $A 20$ gene belongs to the zinc-finger protein family. Its product can effectively enhance the ability of ECs to resist the damage caused by pathogenic agents and inhibit the pathological proliferation of SMCs. In addition, it downregulates the expression of some inflammatory mediators, thereby inhibiting the inflammatory response. We previously modified seed cells with $A 20$ and effectively constructed tissue-engineered blood vessels with anti-atherosclerosis potential and demonstrated the therapeutic effects of these engineered blood vessels in animal experiments. ${ }^{18}$ The latter studies indicated that the $A 20$ gene has the potential to inhibit atherosclerosis plaque formation and prevent restenosis.

In this study, we first locally delivered the $A 20$ gene into the vascular wall via eluting stents and observed its effect on the prevention of restenosis of the carotid artery in a porcine model. The present study was aimed at exploring a new avenue for the prevention and treatment of carotid artery restenosis.

\section{Materials and methods Stents}

Under sterile conditions, type I cowhide collagen (Kensey Nash Corporation, Exton, PA, USA) was added to the crosslinking agent ethyl-dimethyl-amino-propyl carbodiimide (Sigma-Aldrich Co., StLouis, MO, USA) (0.1 mg/mg collagen). A bare-metal stent (provided by Shanghai Weichuang Company, Ltd., Shanghai, People's Republic of China) was dipped into the collagen solution and dried at $37^{\circ} \mathrm{C}$. The latter steps were repeated several times until each stent was coated with $\sim 1 \mathrm{mg}$ of collagen. The stents were then examined under a scanning electron microscope to ensure proper collagen coating. The collagen-coated stents were placed in an $\mathrm{N}$-succinimidyl-3-(2-pyridyldithiol) propionate (SPDP; Pierce, Holmdel, USA) solution ( $20 \mathrm{mmol} / \mathrm{L})$ at room temperature for 2 hours. SPDP-treated stents were rinsed with phosphate-buffered saline (PBS) and then reacted with dithiothreitol (Beyotime, Shanghai, People's Republic of China) at room temperature for 0.5 hours followed by five times rinsing with PBS. Stents were then placed at room temperature overnight in a solution containing a mouse monoclonal antibody to bovine DNA (US Biologicals, Swampscott, USA) that had been reacted with SPDP and purified (IgM-SPDP). The antibody-bound stents were fully rinsed with PBS and then placed into a pCDNA3.1EHA20 (with E-tag sequence: GGT GCG CCGGTG CCG TAT CCG GAC CCG CTG GAA CCG CGT) solution or a pCDNA3.1 void vector solution $(100 \mu \mathrm{L}$ PBS containing $100 \mu \mathrm{g}$ plasmid DNA $),{ }^{18,19}$ incubated at $37^{\circ} \mathrm{C}$ for 1 hour, and then rinsed five times with PBS. Fifteen microliters of ESCORT liposome transfection reagent (Hoffman-La Roche Ltd., Basel, Switzerland) was added to the solution, and the treated stents were incubated at room temperature for 15 minutes before use in subsequent experiments. Bare-metal stents were used as controls.

\section{In vitro transfection}

To test whether the $A 20$ gene carried by the stents could be transfected into ECs in vitro, the following procedures were carried out. Prior to the study, all patients were informed regarding the procedure and its possible complications, and agreed to participate. The Ethics Committee of the Third Military Medical University approved this study. Under sterile conditions, $15-20 \mathrm{~cm}$ of human umbilical cord was collected, washed with D-Hank's solution, then perfused with $0.125 \%$ trypsin, and digested at $37^{\circ} \mathrm{C}$ for 20 minutes. The digestion was terminated with M199 culture medium containing $20 \%$ fetal calf serum (FCS). The digestion mixture was centrifuged at 1,000 rpm for 8 minutes, and the supernatant fluid was discarded. The recovered cells were resuspended in M199 medium containing 20\% FCS, and inoculated at a concentration of $1 \times 10^{5} / \mathrm{mL}$. The culture medium was M199 solution containing $20 \% \mathrm{FCS}, 90 \mathrm{mg} / \mathrm{mL}$ sodium heparin, and $25 \mathrm{mg} / \mathrm{L}$ endothelial growth factor. The cells were cultured in an incubator with $5 \% \mathrm{CO}_{2}$ at $37^{\circ} \mathrm{C}$.

The pCDNA3.1EHA20-bound stents were placed into a six-well plate, and $1 \mathrm{~mL}$ of ECs was added into each well at a concentration of $1 \times 10^{5} / \mathrm{mL}$ and incubated at $37^{\circ} \mathrm{C}$ for 2 hours. Stents were then rotated by $120^{\circ}$ along the long axis, 
and the cell suspension was added to each well at the same concentration. These steps were repeated thrice. After 7 days of culture, stents were fixed with $4 \%$ paraformaldehyde for 30 minutes, and E-tag monoclonal antibody (Amersham Pharmacia Biotech Ltd., Shanghai, People's Republic of China) was added and incubated at room temperature for 30 minutes. Phosphate buffer was used to wash away the excess monoclonal antibody, followed by addition of fluorescein isothiocyanate-labeled secondary antibody and staining of the nuclei with 4',6-diamidino-2-phenylindole (DAPI). Stents were observed using a laser confocal microscope (Leica Microsystems, Wetzlar, Germany), with blue fluorescence indicating DAPI-labeled nuclei of ECs and green fluorescence indicating ECs transfected with $A 20$.

\section{Animals}

The animal study was in conformity with national regulations and carried out after obtaining permits for the use of animals issued by the Ministry of Health. The animal use and experimental protocols were reviewed and approved by the Ethics Committee of Third Military Medical University. A total of 35 miniature pigs (12-14 weeks old), with a weight of $25-27 \mathrm{~kg}$, were purchased from the Center of Laboratory Animals, Third Military Medical University (Chongqing, People's Republic of China) and were fed in a standard manner. Pigs were randomly divided into three groups: pCDNA3.1EHA20 $(n=12)$, CDNA3.1 void vector $(n=12)$, and bare-metal stent $(n=11)$. From 3 days before surgery to the follow-up period, all animals were orally administered $300 \mathrm{mg}$ of aspirin and $75 \mathrm{mg}$ of clopidogrel every day. The animals were anesthetized with $25 \mathrm{mg} / \mathrm{kg}$ sodium pentobarbital, followed by tracheal intubation and mechanical ventilation. The animals were sacrificed 3 days, 2 weeks, and 3 months after surgery.

\section{Porcine model of carotid artery stent}

Three kinds of stents (pCDNA3.1EHA20, pCDNA3.1 void vector, and bare-metal stent) were randomly placed in the left and right carotid artery of each pig. Briefly, an 8Fr arterial sheath was inserted from the right femoral artery, and an 8Fr-guiding catheter was inserted into the carotid artery. A 0.014 in. guide wire was sent to the distal end of the carotid artery. The stents were advanced along the guide wire to the target vessel's location and were placed at a balloon-to-vessel ratio of 1.2:1 by angiography. After the placement of the stents, angiography was performed to determine whether the lumen was unobstructed and whether filling defects, vascular wall dissection, embolization, stent migration, or remote vasospasm was present. Regular anti-infection treatment was employed after the surgery. The skin around the wound surface was disinfected routinely, washed with sterile normal saline, and dried. The animals were bred in a good environment. Subsequent follow-up angiography was carried out through catheterization of the contralateral femoral artery.

\section{Analysis of $A 20$ expression}

Three days after stent placement, proteins were extracted from the pig carotid arteries. E-tag and $A 20$ expression was confirmed by Western blot analysis, as described previously. ${ }^{20}$ Electrophoresis, membrane transfer, and signal development were performed. Tissue lysates were separated under reducing conditions by SDS-PAGE (Bio-Rad Laboratories Inc., Hercules, CA, USA), and transferred to polyvinylidenefluoride membranes (PerkinElmer Inc., Waltham, MA, USA) by semidry electroblotting. Membranes were probed with mouse anti-E-tag antibody (Amersham Pharmacia Biotech), mouse anti-human $A 20$ antibody (1/1,000; Oncogene, NY, USA), and mouse antiglyceraldehyde3-phosphate dehydrogenase (EMD Millipore, Billerica, MA, USA). The blots were washed and exposed to horseradish peroxidaseconjugated antimouse IgG secondary antibody (Santa Cruz Biotechnology Inc., Dallas, TX, USA) and then developed using the enhanced chemiluminescence reagent (PerkinElmer Inc.). The results were normalized against glyceraldehyde3-phosphate dehydrogenase gene expression.

\section{Scanning electron microscopy}

Stents were removed after either 14 days or 3 months in vivo, rinsed with physiological saline, fixed with $3 \%$ glutaraldehyde, and then cut open along the longitudinal axis. A scanning electron microscope (Model JSM 5510; JEOL, Tokyo, Japan) was used to examine the intimal layer. Photomicrographs of each specimen taken using scanning electron microscopy (SEM) were examined using a computer-assisted digital assessment system. The percent area of re-endothelialization compared with the total luminal surface area was calculated. For each specimen, five photomicrographs were taken at $\times 400$ magnification using SEM, and the re-endothelialization area for each artery was represented by the sum of the data for the five photographed subareas. ${ }^{21}$

Stent endothelialization score was defined as the extent of the circumference of the arterial lumen covered by ECs and graded from 1 to $3(1=25 \%, 2=25 \%-75 \%$, and $3 \geq 75 \%)$. ${ }^{22}$

\section{Morphological measurement and analysis}

Extracted blood vessels were embedded and sectioned as described earlier, and stained with hematoxylin and eosin. 
All sections were observed under an optical microscope (DFC300FX; Leica Microsystems) for intimal hyperplasia and vascular wall damage. Data analysis was carried out by use of an Image-Pro Plus computer-assisted digital system (Leica Qwin Plus V3.2.1; Leica Microsystems). Stent area and the lumen area (LA) were calculated, and the neointimal area (NA) was calculated using the formula (strut area luminal area). Percentage stenosis area was calculated as $(1-$ luminal area/strut area) $\times 100 \%$.

\section{Evaluation of arterial injury and inflammation scores}

The severity of arterial injury was scored as previously described by Schwartz et $\mathrm{al}^{23}$ ( $0=$ no injury; $1=$ break in the internal elastic membrane; $2=$ perforation of the media; and $3=$ perforation of the external elastic membrane to the adventitia). The inflammation score for each individual strut was graded according to the following criteria: ${ }^{22,24} 0=$ no inflammatory cells surrounding the strut; $1=$ light noncircumferential lymphohistiocytic infiltrate surrounding the strut; $2=$ localized, moderate to dense cellular aggregate surrounding the strut non-circumferentially; and $3=$ circumferential, dense lymphohistiocytic cell infiltration of the strut. Arterial injury and inflammation scores for each cross-section were calculated by dividing the sum of the individual injury and inflammation scores by the total number of struts at the examined section, as previously described.

\section{Assessment of inflammatory cytokines after stent implantation}

Peripheral blood samples were drawn before stent implantation and 3 days after stent implantation in the carotid artery. To limit circadian variation in cytokine production, fasting blood samples were collected in the morning into pyrogenfree tubes. Within 30 minutes of blood collection, plasma was obtained after a centrifugation at $3,000 \mathrm{rpm}$ at $4^{\circ} \mathrm{C}$ for 15 minutes and stored at $-80^{\circ} \mathrm{C}$ until assay. Plasma cytokine concentrations were measured by commercial enzyme-linked immunosorbent assay for interleukin-1 $\beta$ (IL-1 $\beta$ ) and tumor necrosis factor- $\alpha$ (TNF- $\alpha$ ) according to manufacturer's instructions (R\&D Systems, Inc., Minneapolis, MN, USA). Measurements were performed in duplicate, and the mean value of two measurements was used.

\section{Statistical analysis}

All values are expressed as mean \pm standard deviation. Significance of variability among the means of the experimental groups was determined by one-way analysis of variance, using SPSS 11.0 software. $P<0.05$ was considered as statistically significant.

\section{Results \\ Coated stents}

The stents were examined under a scanning electron microscope. The surface of bare-metal stents was smooth without any attachments (Figure 1A). The processed stents were evenly covered with a layer of collagen on their surface (Figure 1B).

\section{Evaluation of in vitro transfection}

Cultured ECs were seeded on the stents carrying A20. The transfection of these cells was evaluated by examination under a confocal laser scanning microscope. Figure $1 \mathrm{C}$ and D shows the general morphology of a stent in white balance mode. DAPI-stained nuclei appeared in blue (Figure 1E and F), indicating that cells were evenly distributed on the stent, covering it almost fully. Some of the cells emitted green fluorescence, suggesting that they were transfected with $A 20$ (Figure $1 \mathrm{G}$ ), while they were not transfected with A20 (Figure 1H).

\section{Animals}

Stents were successfully implanted in all animals. Angiography was performed 15 minutes later to confirm that the lumen was unobstructed, without filling defects, vascular wall dissection, embolization, stent migration, or distal vasospasm. No significant differences were observed in heart rate, blood pressure, and body weight between three groups in preoperative, intraoperative, and follow-up angiography. There were no obvious abnormalities in behavior, eating, and bowel movement after surgery.

\section{Detection of $A 20$ protein expression}

Samples were collected from carotid arteries 3 days after they had been implanted with stents. Proteins were extracted from the collected blood vessel samples. Western blot analysis showed that there was no E-tag and $A 20$ protein in the pCDNA3.1 void vector and bare-metal group (Figure 2A and B). There were E-tag and $A 20$ protein expression in the pCDNA3.1EHA20 group (Figure 2C).

\section{Detection of stent re-endothelialization}

To determine the effect of three kinds of stents on reendothelialization, stent coverage by ECs was evaluated by SEM. Two weeks after stent placement, there was a 

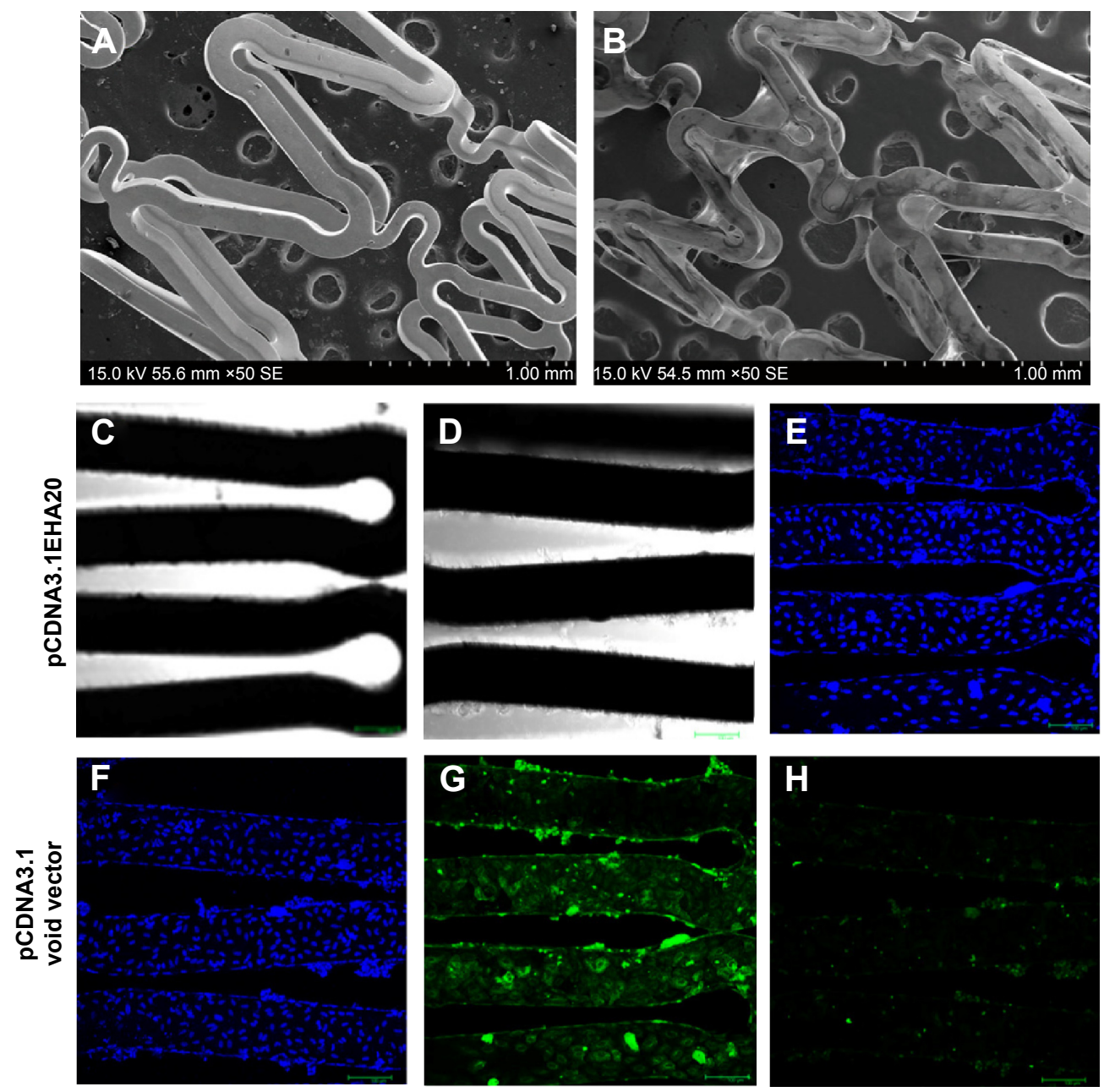

Figure I Observation on the surface of the stent.

Notes: Stent surface under the scanning electron microscope. (A) Bare-metal stent. (B) Processed stent covered with a layer of collagen. Transfection of cells on the stent under confocal laser scanning microscope. (C and $\mathbf{D})$ General morphology of a stent in white balance mode. (E and $\mathbf{F})$ The nuclei of the endothelial cells on a stent were stained blue with DAPI. (G) Some A20-transfected cells are shown in green. $(\mathbf{H})$ No cells are shown in green. Scale bars $(\mathbf{C}-\mathbf{H})=\sim 500 \mu \mathrm{m}$.

Abbreviation: DAPI, 4',6-diamidino-2-phenylindole.

significantly higher extent of endothelial coverage above struts in the pCDNA3.1EHA20 group $(85.10 \% \pm 5.47 \%)$ compared with pCDNA3.1 void vector group $(63.10 \% \pm 3.93 \%)$ or bare-metal stent group $(85.10 \% \pm 5.47 \%)(P<0.05$, Figure 3). There were no differences in the percentage of re-endothelialization between the pCDNA3.1 void vector group and bare-metal stent group ( $P>0.05$, Figure 3$)$.

Three months after stent placement, the re-endothelialization areas for all stents were higher, there were no differences in the percentage of re-endothelialization $(P>0.05)$, and the ECs completely covered the blood vessel in three groups (Figure 4).

\section{Histomorphometric analysis}

There was no significant difference in arterial injury score and inflammation score among the three stent groups at 14 days and 3 months ( $P>0.05$, Figure 5). Three months after stent implantation, morphological measurement results were as follows: 1) internal elastic lamina area $\left(\mathrm{mm}^{2}\right)$ : no significant difference among the three groups; 2) LA $\left(\mathrm{mm}^{2}\right)$ : the baremetal stent group and pCDNA3.1 void vector group had significantly smaller LA than the pCDNA3.1EHA20 group $(P<0.01)$; 3) NA $\left(\mathrm{mm}^{2}\right)$ : the bare-metal stent group and pCDNA3.1 void vector group had significantly larger NA than the pCDNA3.1EHA20 group $(P<0.01)$; and 4$)$ arterial stenosis rate (\%): the bare-metal stent group and pCDNA3.1 void vector group had significantly larger stenosis rate than the pCDNA3.1EHA20 group $(P<0.01)$. There was no significant difference in the above between the bare-metal stent group and pCDNA3.1 void vector group $(P>0.05)$. (Refer to the specific values in Table 1, and typical pathological changes in Figure 6.) 


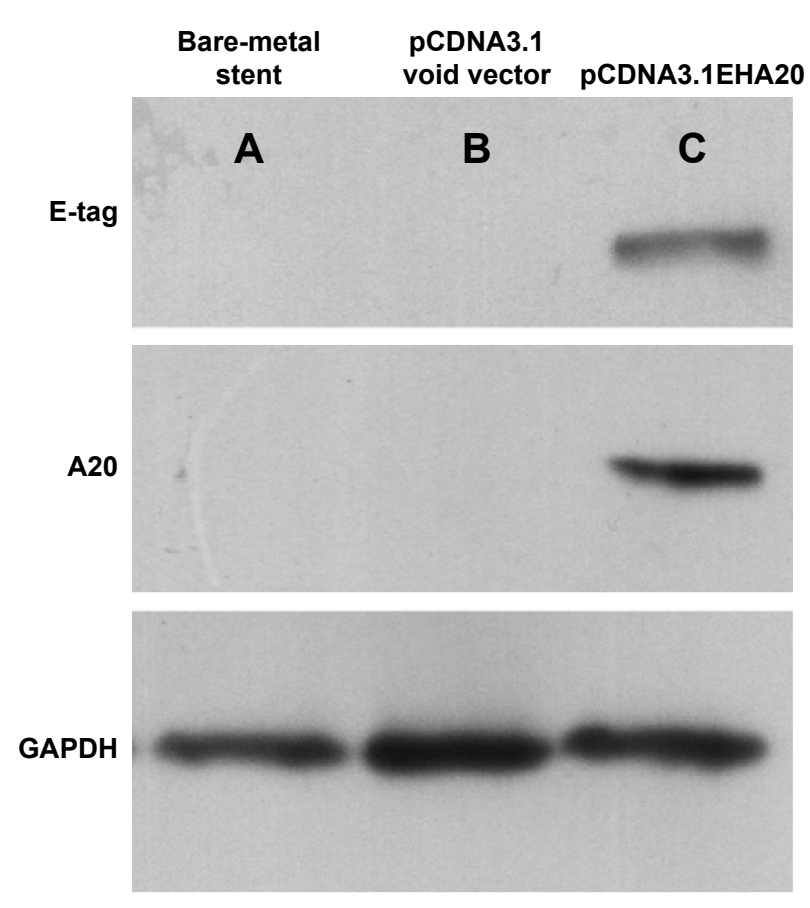

Figure 2 Western blot analysis.

Notes: (A) No E-tag and A20 protein expression are seen in the bare-metal stent group. (B) No E-tag and A20 protein expression are seen in the PCDNA3.I void vector group. (C) E-tag and A20 protein expression are seen in the PCDNA3. IEHA20 group.

\section{Expression of inflammatory markers after stent implantation}

We used enzyme-linked immunosorbent assay to determine the plasma levels of IL- $1 \beta$ and TNF- $\alpha$ before stent implantation and 3 days after stent implantation. TNF- $\alpha$ and IL- $1 \beta$ levels were not significantly different between the three groups before stent implantation. Their levels were significantly lower in the pCDNA3.1EHA20 group compared with the bare-metal stent group and the pCDNA3.1 void vector group $(P<0.05$, Figure 6$)$. These results indicate that pCDNA3.1EHA20 coating on the stent downregulated inflammatory mediator expression and inhibited the inflammatory reaction.

\section{Discussion}

We have studied the possibility of using A20-modified intravascular stents to reduce neointimal formation and accelerate re-endothelialization as a treatment strategy. The objective of this study was to develop a treatment strategy that could reduce the incidence of ISR without increasing the risk of thrombosis. We used collagen to cover stents and then modified the stents with the $A 20$ gene. Results showed
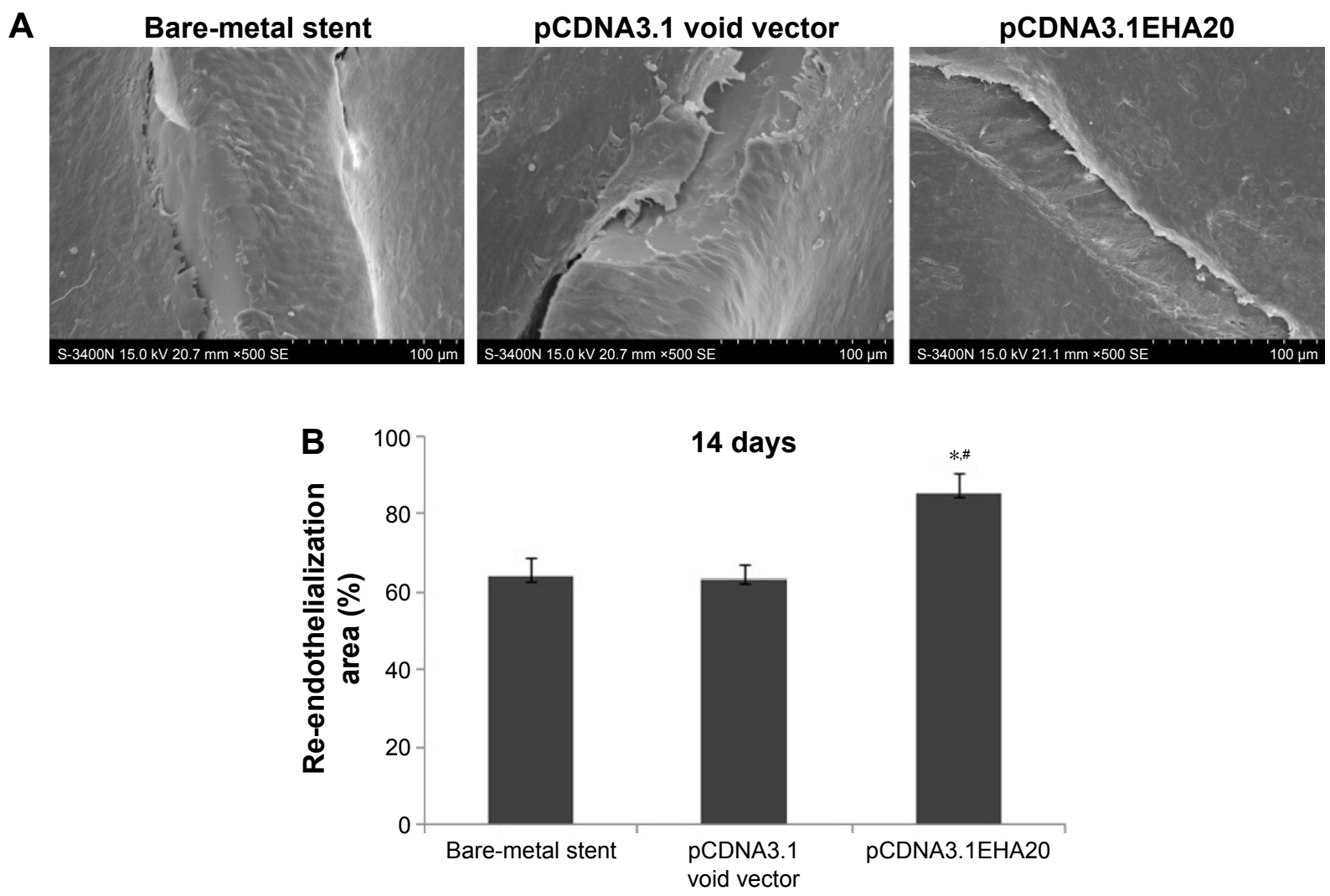

Figure 3 Re-endothelialization of arteries assessed by scanning electron microscopy at 14 days post stent implantation.

Notes: (A) Typical scanning electron microscopy images of the intact stented arterial segments. (B) Percentage of re-endothelialized area of the total stented area. There was significantly higher re-endothelialized area in the pCDNA3. IEHA20 group compared with bare-metal stent group and the $p C D N A 3$.I void vector group. $* P<0.05$ vs bare-metal stent group; ${ }^{\# P}<0.05$ vs $P C D N A 3$.I void vector group. 
A

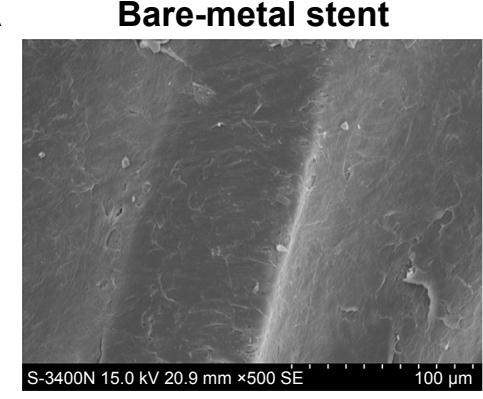

pCDNA3.1 void vector

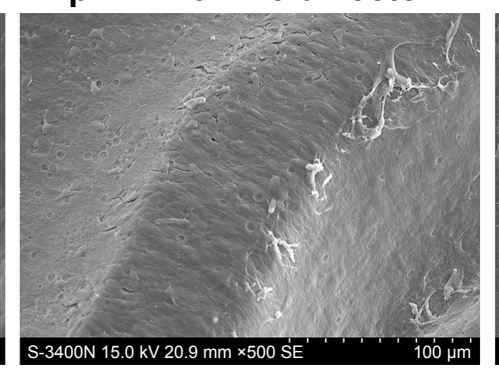

PCDNA3.1EHA20

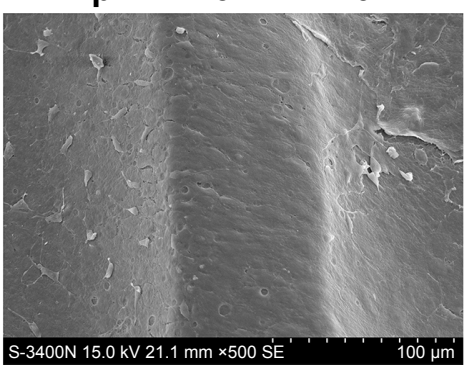

B

3 months

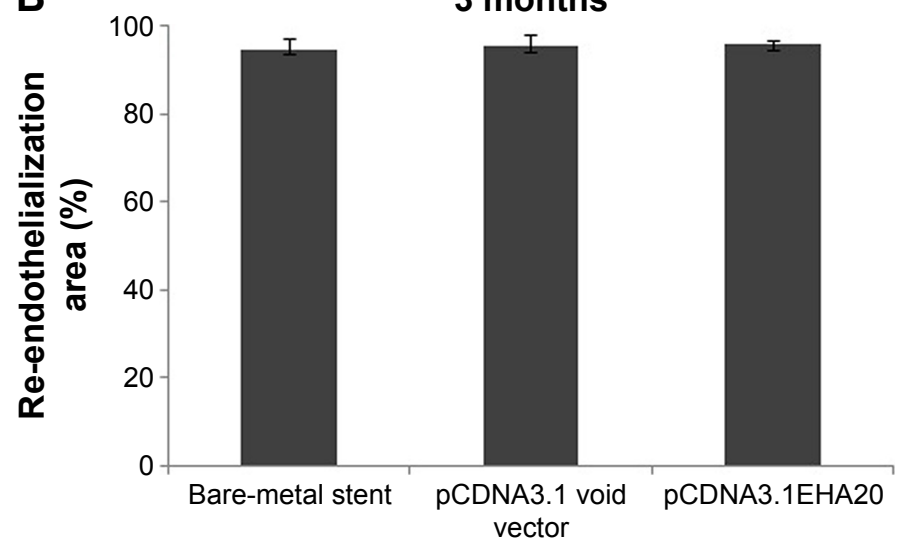

Figure 4 Re-endothelialization of arteries assessed by scanning electron microscopy at 3 months post stent implantation.

Notes: (A) Typical scanning electron microscopy images of the intact stented arterial segments. (B) Percentage of re-endothelialized area of total stented area. There were no differences in the percentage of re-endothelialization among the three groups $(P>0.05)$.
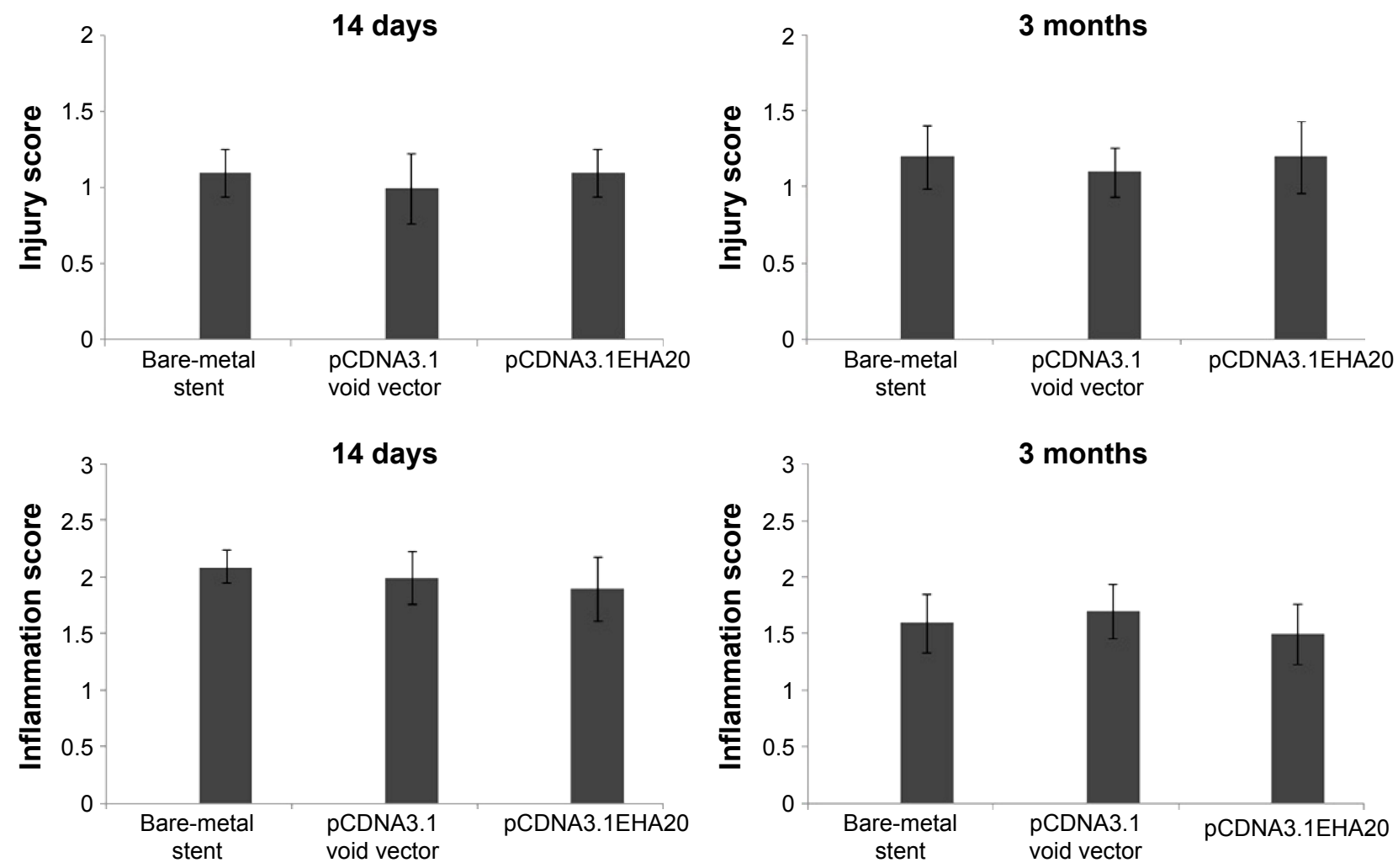

Figure 5 Quantification of artery injury and inflammation scores around the stent struts at 14 days and 3 months after stent implantation.

Notes: There was no significant difference in arterial injury score and inflammation score among the three stent groups at 14 days and 3 months $(P>0.05)$. 
Table I Histomorphological measurements at 3 months

\begin{tabular}{lllll}
\hline Group & IEL $\left(\mathbf{m m}^{2}\right)$ & LA $\left(\mathbf{m m}^{2}\right)$ & NA $\left(\mathbf{m m}^{2}\right)$ & AS $(\%)$ \\
\hline Bare-metal stent & $12.52 \pm 0.38$ & $6.23 \pm 0.35$ & $6.32 \pm 0.59$ & $52.03 \pm 3.75$ \\
PCDNA3.I void vector & $12.16 \pm 0.31$ & $6.03 \pm 0.33$ & $6.13 \pm 0.57$ & $50.37 \pm 3.60$ \\
pCDNA3. IEHA20 & $12.09 \pm 0.24$ & $9.52 \pm 0.27^{*}$ & $2.58 \pm 0.35^{*}$ & $21.27 \pm 2.58^{*}$ \\
\hline
\end{tabular}

Notes: *There is a statistically significant difference compared with the bare-metal stent or $\mathrm{PCDNA3.I}$ void vector group $(P<0.0 \mathrm{I})$. Data presented as mean \pm standard deviation.

Abbreviations: IEL, internal elastic lamina area; LA, lumen area; NA, neointimal area; AS, arterial stenosis rate.

that compared with stents in the control group, the $A 20$-genemodified stents significantly accelerated re-endothelialization in the blood vessels, which was apparent at day 14 and persisted at 3-month examinations. This effect was accompanied by a clear reduction of neointimal formation as detected by histomorphological and quantitative angiographic analysis.
Therefore, for the first time, to our knowledge, we have proved that $A 20$-gene-modified intravascular stents can reduce restenosis while promoting $\mathrm{EC}$ regeneration in a porcine carotid artery model.

Although the use of DES has significantly reduced restenosis, it has been found to be associated with long-term

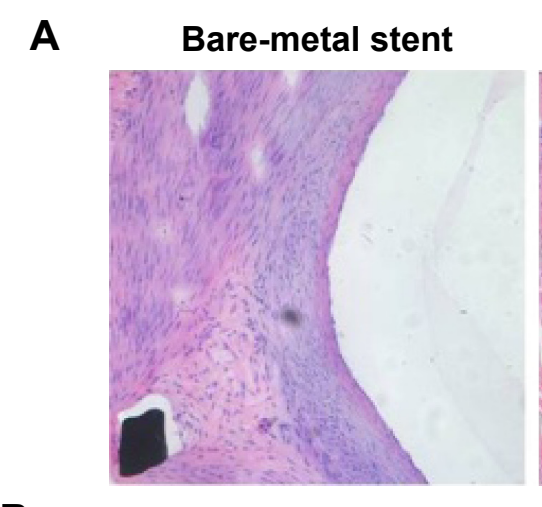

B
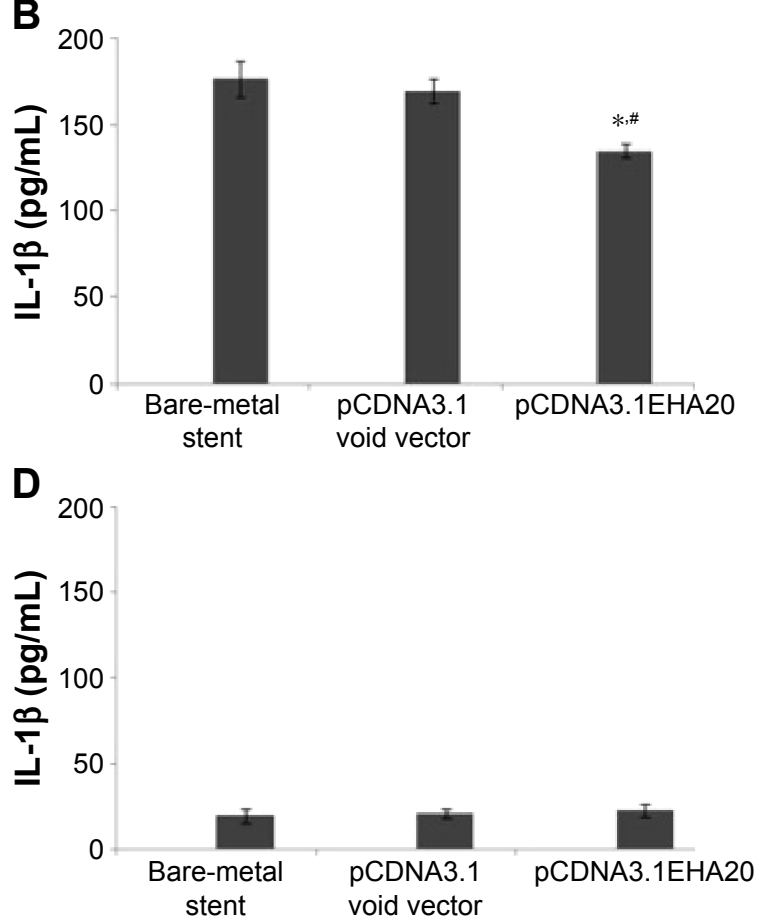

pCDNA3.1 void vector

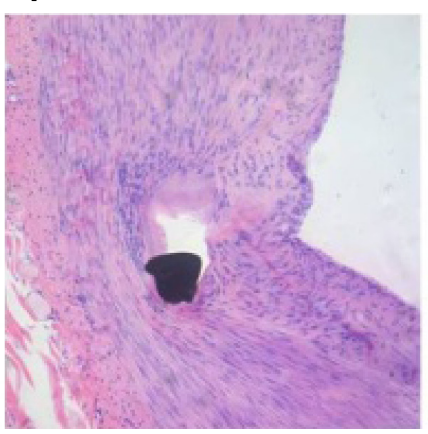

C

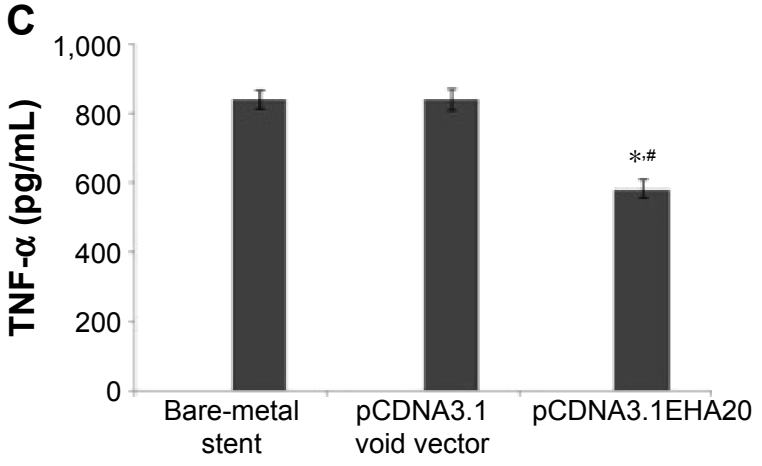

E

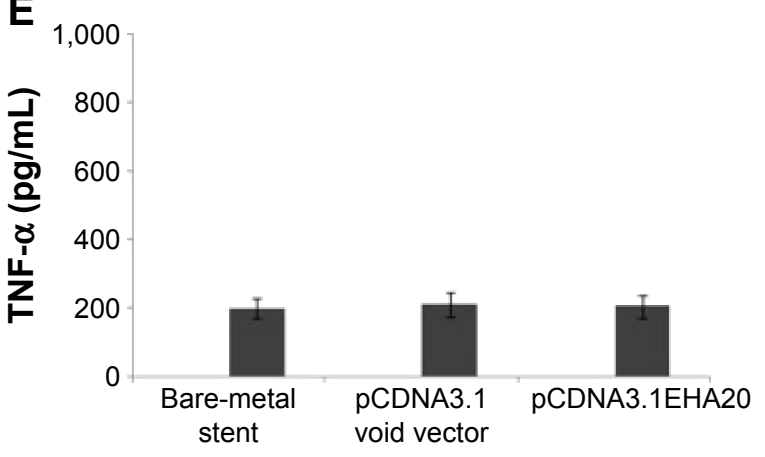

Figure 6 Typical images of stented carotid arteries and expression of inflammatory markers.

Notes: $(A)$ H\&E staining $(\times 200)$ showed obvious intimal hyperplasia in the bare-metal stent group and the pCDNA3.I void vector group and relatively mild intimal hyperplasia in the PCDNA3.IEHA20 group. (B) Plasma levels of IL-I $\beta$ after stent implantation with ELISA. (C) Plasma levels of TNF- $\alpha$ after stent implantation with ELISA. (D and E) TNF- $\alpha$ and IL-I $\beta$ level were not significantly different between the three groups before stent implantation $* P<0.05$ vs bare-metal stent group; ${ }^{*}<<0.05$ vs PCDNA3.I void vector group.

Abbreviations: H\&E, hematoxylin and eosin; IL-I $\beta$, interleukin-I $\beta$; ELISA, enzyme-linked immunosorbent assay; TNF- $\alpha$, tumor necrosis factor- $\alpha$. 
stent thrombosis. Recent reports indicate that after the discontinuation of clopidogrel administration, DES increases long-term mortality and Q-wave infarction rates, compared with bare-metal stents. ${ }^{11,25,26}$ Reducing restenosis while promoting re-endothelialization could be an alternative strategy to reduce stent thrombosis. Further benefits include avoiding the harmful effects of noncontinuous use of anti-platelet drugs. Compared with DES, gene-modified stents have the benefit of the combination of angioplasty and local gene delivery, ${ }^{27}$ for which the screening of appropriate functional genes is particularly important.

During their research on TNF response genes in ECs, Dixit et $\mathrm{al}^{28}$ revealed that the $A 20$ gene has an open reading frame that encodes a novel zinc-finger protein. Currently, studies have shown that $A 20$ binds to TRAF through its $\mathrm{N}$-terminus, and binds to ABIN and IKK- $\gamma$ via its C-terminus, thereby exerting its regulatory effects on the activity of nuclear factor $\mathrm{\kappa B}$ and $\mathrm{AP}-1 .{ }^{29} \mathrm{On}$ the one hand, $A 20$ downregulates their activity levels, while on the other it also disrupts their synergistic regulatory effects on inflammatory response, exhibited as downregulation of the expression of inflammatory mediators such as TNF- $\alpha$, IL-1, IL-6, and IL- 8 and the inhibition of TNF- $\alpha$-induced apoptosis. ${ }^{30}$ This will ultimately limit inflammation in the body and protect the cells from damage. Gene knockout experiments have shown that the $A 20$ gene is an indispensable functional gene for the human body to resist inflammation, as well as an important gene for the physiological protection of ECs. $A 20$ gene counteracts TNF, FAS, and natural killer cell-mediated cell death, and effectively blocks the NF- $\kappa B$ and the TOLL signaling pathways. ${ }^{31,32}$

Overexpression of the $A 20$ gene prevents atherosclerosis in vascular grafts in allogenic kidney transplantation. In the ApoE $^{-/-}$mouse model, A20 could effectively inhibit the occurrence of atherosclerosis. ${ }^{33}$ Furthermore, A20 protein overexpression prevented neointimal hyperplasia following rat carotid balloon injury and reverted pre-existing lesions. ${ }^{34,35}$

Previously, we effectively constructed tissue-engineered blood vessels with anti-atherosclerosis potential using $A 20$ modified seed cells. Animal experiments demonstrated the therapeutic effects of these engineered blood vessels. ${ }^{18}$ Therefore, based on the dual function of $A 20$ in inhibiting inflammatory response and protecting ECs, we used $A 20$ to modify intravascular stents. The modified stents were implanted in vivo and investigated for their therapeutic effects and related mechanisms.

To accomplish this treatment strategy, it was necessary to identify an ideal stent/polymer/gene combination. Thus, we explored how to improve gene adherence to the stent. Because SPDP can effectively link two proteins without affecting their activities, ${ }^{36}$ it was used to link anti-DNA antibodies to collagen, ${ }^{37}$ thereby firmly attaching the $A 20$ gene to the stents by taking advantage of antigen-antibody binding and allowing the $A 20$ gene to withstand the impact of certain physiological conditions without falling off from the stents and to reach its maximum effectiveness at the target site. In addition, we chose liposome as the transfection reagent to minimize the adverse effects of other transfection reagents on cells. We first performed in vitro experiments, culturing the ECs on the stents carrying A20. Subsequent examination showed that $A 20$ could be transfected into ECs. Further animal experiments showed that $A 20$ protein expression could be detected 72 hours after stent implantation, indicating that this method can be used to successfully deliver genes to the vascular wall. Furthermore, we found that the $A 20$ gene product downregulated the expression of inflammatory mediators such as TNF- $\alpha$, IL-1 and inhibited the inflammatory reaction.

Our findings indicate that the effects of A20-modified stent could be seen on day 14. First, the degree of re-endothelialization was significantly higher in the experimental group than in the control group, indicating that A20-modified stent protected ECs. After 3 months, the results became even more evident. Although both the control group and the experimental group exhibited reendothelialization, intimal hyperplasia was significantly reduced in the experimental group. Therefore, it is evident that $A 20$ exerts its effects through protecting ECs and inhibiting proliferation of SMCs, which ultimately prevents the development of restenosis. Further studies are necessary to determine the mechanisms underlying these different processes.

Although vascular gene therapy is shown to be feasible in animal models, there is no definitive evidence on its benefits in humans. This may be due to many potential factors, one of which is the delivery method of the therapeutic genes to the blood vessel wall. Stent-based, liposome-mediated gene delivery has rarely been studied. However, the application of intravascular stents as carriers of therapeutic genes can extend the duration of the effect and achieve targeted delivery, which will generate ideal effects and have great application prospects. This study is among the very few reports on stent-based gene delivery in the vascular wall to reduce intimal hyperplasia and promote $\mathrm{EC}$ regeneration in a porcine model. It is also the first report to elucidate the effect of stent-based delivery of the $A 20$ gene on the vascular wall. 


\section{Conclusion}

Our studies have proved that stent-based overexpression of A20 inhibits ISR and promotes endothelial regeneration at the location of vascular lesions. This could be an ideal strategy to address the problem of ISR and provide an alternative to current DESs.

\section{Acknowledgments}

This work was supported by the National Natural Science Foundation of China for Distinguished Young Scholars (no 81000671), Natural Science Foundation of ChongQing (no 2010BB5190), and the National Natural Science Foundation of China (no 81471194). The authors also thank Ms Lu Ju for her assistance in SEM and confocal microscopic analysis.

\section{Disclosure}

The authors report no conflicts of interest in this work.

\section{References}

1. Demaerschalk BM, Hwang HM, Leung G. US cost burden of ischemic stroke: a systematic literature review. Am J Manag Care. 2010; 16(7):525-533.

2. Rothwell PM, Algra A, Amarenco P. Medical treatment in acute and long-term secondary prevention after transient ischaemic attack and ischaemic stroke. Lancet. 2011;377(9778):1681-1692.

3. Bonati LH, Dobson J, Featherstone RL, et al. Long-term outcomes after stenting versus endarterectomy for treatment of symptomatic carotid stenosis: the International Carotid Stenting Study (ICSS) randomised trial. Lancet. 2015;385(9967):529-538.

4. Mantese VA, Timaran CH, Chiu D, Begg RJ, Brott TG; CREST Investigators. The carotid revascularization endarterectomy versus stenting trial (CREST): stenting versus carotid endarterectomy for carotid disease. Stroke. 2010;41(10):S31-S34.

5. Brott TG, Hobson RW, Howard G, et al. Stenting versus endarterectomy for treatment of carotid-artery stenosis. N Engl J Med. 2010; 363(1):11-23.

6. Bonati LH, Dobson J, Algra A, et al. Short-term outcome after stenting versus endarterectomy for symptomatic carotid stenosis: a preplanned meta-analysis of individual patient data. Lancet. 2010; 376(9746):1062-1073.

7. Shin YS, Kim BM, Suh SH, et al. Wingspan stenting for intracranial atherosclerotic stenosis: clinical outcomes and risk factors for in-stent restenosis. Neurosurgery. 2013;72(4):596-604.

8. Wasser K, Schnaudigel S, Wohlfahrt J, et al. Clinical impact and predictors of carotid artery in-stent restenosis. J Neurol. 2012;259(9): 1896-1902.

9. Morice MC, Serruys PW, Sousa JE, et al. A randomized comparison of a sirolimus-eluting stent with a standard stent for coronary revascularization. N Engl J Med. 2002;346(23):1773-1780.

10. Kedhi E, Joesoef KS, McFadden E, et al. Second-generation everolimuseluting and paclitaxel-eluting stents in real-life practice (COMPARE): a randomised trial. Lancet. 2010;375(9710):201-209.

11. Shuchman M. Debating the risks of drug-eluting stents. N Engl J Med. 2007;356(4):325-328.

12. Stone GW, Moses JW, Ellis SG. Safety and efficacy of sirolimus and paclitaxel eluting coronary stents. N Engl J Med. 2007;356(10):998-1008.

13. Hharif F, Hynes SO, Cooney R, et al. Gene-eluting stents: adenovirusmediated delivery of eNOS to the blood vessel wall accelerates re-endothelialization and inhibits restenosis. Mol Ther. 2008;16(10): 1674-1680.
14. Liu J, Xu Y, Yang Q, et al. Reduction biodegradable brushed PDMAEMA derivatives synthesized by atom transfer radical polymerization and click chemistry for gene delivery. Acta Biomater. 2013; 9(8):7758-7766.

15. Sharif F, Hynes SO, McMahon J, et al. Gene-eluting stents: comparison of adenoviral and adeno-associated viral gene delivery to the blood vessel wall in vivo. Hum Gene Ther. 2006;17(7):741-750.

16. Bonta PI, Pols TW, van Tiel CM, et al. Nuclear receptor Nurr1 is expressed in and is associated with human restenosis and inhibits vascular lesion formation in mice involving inhibition of smooth muscle cell proliferation and inflammation. Circulation. 2010;121(18): 2023-2032.

17. Takahashi M. Genetic susceptibility to restenosis: role of bone marrow cells and inflammatory response. Arterioscler Thromb Vasc Biol. 2009; 29(10):1407-1408.

18. Zhu CH, Ying DJ, Mi JH, et al. Development of anti-atherosclerosis tissue engineering blood vessel using zinc finger protein A20 regulated endothelial progenitor cells. Biomaterials. 2008;29(17): 2628-2636.

19. Hou CL, Zhang W, Wei Y, et al. Zinc finger protein A20 overexpression inhibits monocyte homing and protects endothelial cells from injury induced by high glucose. Genet Mol Res. 2011;10(2):1050-1059.

20. Zhu $\mathrm{CH}$, Ying DJ, Mi JH, et al. The zinc finger protein A20 gene protects endothelial cell from burns serum injury. Burns. 2004;30(2): $127-133$.

21. Ma X, Hibbert B, Dhaliwal B, et al. Delayed re-endothelialization with rapamycin-coated stents is rescued by the addition of a glycogen synthase kinase-3beta inhibitor. Cardiovasc Res. 2010;86(2): 338-345.

22. Carter AJ, Aggarwal M, Kopia GA, et al. Long-term effects of polymerbased, slow-release, sirolimus-eluting stents in a porcine coronary model. Cardiovasc Res. 2004;63(4):617-624.

23. Schwartz RS, Huber KC, Murphy JG, et al. Restenosis and the proportional neointimal response to coronary artery injury: results in a porcine model. J Am Coll Cardiol. 1992;19(2):267-274.

24. Steigerwald K, Merl S, Kastrati A, et al. The pre-clinical assessment of rapamycin-eluting, durable polymer-free stent coating concepts. Biomaterials. 2009;30(4):632-637.

25. Lassen JF. Long-term safety and efficacy of drug-eluting stents. Lancet. 2011;377(9773):1213-1214.

26. Cook S, Ladich E, Nakazawa G, et al. Correlation of intravascular ultrasound findings with histopathological analysis of thrombus aspirates in patients with very late drug-eluting stent thrombosis. Circulation. 2009;120(5):391-399.

27. Fishbein I, Alferiev I, Bakay M, et al. Local delivery of gene vectors from bare-metal stents by use of a biodegradable synthetic complex inhibits in-stent restenosis in rat carotid arteries. Circulation. 2008; 117(16):2096-2103.

28. Dixit VM, Green S, Sarma V, et al. Tumor necrosis factor-alpha induction of novel gene products in human endothelial cells including a macrophage-specific chemotaxin. J Biol Chem. 1990;265(5): 2973-2978.

29. Heyninck K, Beyaert R. A20 inhibits NF-kappaB activation by dual ubiquitin-editing functions. Trends Biochem Sci. 2005;30(1):1-4.

30. Lee EG, Boone DL, Chai S, et al. Failure to regulate TNF-induced NF-kappaB and cell death responses in A20-deficient mice. Science. 2000;289(5488):2350-2354.

31. Shembade N, Harhaj NS, Parvatiyar K, et al. The E3 ligase Itch negatively regulates inflammatory signaling pathways by controlling the function of the ubiquitin-editing enzyme A20. Nat Immunol. 2008; $9(3): 254-262$.

32. Wertz IE, O'Rourke KM, Zhou H, et al. De-ubiquitination and ubiquitin ligase domains of A20 downregulate NF-kappaB signalling. Nature. 2004;430(7000):694-699.

33. Wolfrum S, Teupser D, Tan M, Chen KY, Breslow JL. The protective effect of A20 on atherosclerosis in apolipoprotein E-deficient mice is associated with reduced expression of NF-kappaB target genes. Proc Natl Acad Sci U S A. 2007;104(47):18601-18606. 
34. Patel VI, Daniel S, Longo CR, et al. A20, a modulator of smooth muscle cell proliferation and apoptosis, prevents and induces regression of neointimal hyperplasia. FASEB J. 2006;20(9):1418-1430.

35. Damrauer SM, Fisher MD, Wada H, et al. A20 inhibits postangioplasty restenosis by blocking macrophage trafficking and decreasing adventitial neovascularization. Atherosclerosis. 2010;211(2): 404-408.
36. Strehblow C, Schuster M, Moritz T, Kirch HC, Opalka B, Petri JB. Monoclonal antibody-polyethyleneimine conjugates targeting Her-2/ neu or CD90 allow cell type-specific nonviral gene delivery. J Control Release. 2005;102(3):737-747.

37. Jin X, Mei L, Song C, et al. Immobilization of plasmid DNA on an antiDNA antibody modified coronary stent for intravascular site-specific gene therapy. J Gene Med. 2008;10(4):421-429.

\section{Publish your work in this journal}

Drug Design, Development and Therapy is an international, peerreviewed open-access journal that spans the spectrum of drug design and development through to clinical applications. Clinical outcomes, patient safety, and programs for the development and effective, safe, and sustained use of medicines are a feature of the journal, which has also been accepted for indexing on PubMed Central. The manuscript management system is completely online and includes a very quick and fair peer-review system, which is all easy to use. Visit http://www.dovepress.com/testimonials.php to read real quotes from published authors.

Submit your manuscript here: http://www.dovepress.com/drug-design-development-and-therapy-journal 